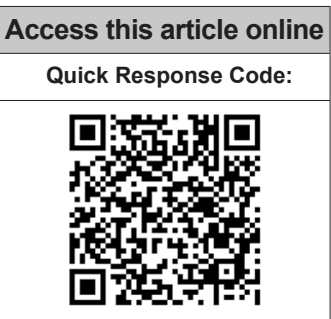

Website:

www.jponline.org

DOI:

10.4103/JLP.JLP_98_17
Department of Pathology,

Dhanalakshmi Srinivasan Medical College and Hospital, Perambalur, Tamil Nadu, India

Address for correspondence: Dr. Pitchaikaran Arul, No 83, Ayyanar Kovil Street, Vinayagampet, Sorapet Post Puducherry - 605 501, India.

E-mail: drarul33@ gmail.com

Submission: 13-06-2017 Accepted: 06-11-2017

\title{
Prevalence and types of preanalytical error in hematology laboratory of a tertiary care hospital in South India
}

\author{
Pitchaikaran Arul, Magesh Pushparaj, Kanmani Pandian, Lingasamy Chennimalai, \\ Karthika Rajendran, Eniya Selvaraj, Suresh Masilamani
}

\section{Abstract:}

BACKGROUND: An important component of laboratory medicine is preanalytical phase. Since laboratory report plays a major role in patient management, more importance should be given to the quality of laboratory tests.

AIM: The present study was undertaken to find the prevalence and types of preanalytical errors at a tertiary care hospital in South India.

MATERIALS AND METHODS: In this cross-sectional study, a total of 118,732 samples ([62,474 outpatient department [OPD] and 56,258 inpatient department [IPD]) were received in hematology laboratory. These samples were analyzed for preanalytical errors such as misidentification, incorrect vials, inadequate samples, clotted samples, diluted samples, and hemolyzed samples.

RESULTS: The overall prevalence of preanalytical errors found was 513 samples, which is $0.43 \%$ of the total number of samples received. The most common preanalytical error observed was inadequate samples followed by clotted samples. Overall frequencies (both OPD and IPD) of preanalytical errors such as misidentification, incorrect vials, inadequate samples, clotted samples, diluted samples, and hemolyzed samples were $0.02 \%, 0.05 \%, 0.2 \%, 0.12 \%, 0.02 \%$, and $0.03 \%$, respectively.

CONCLUSION: The present study concluded that incorrect phlebotomy techniques due to lack of awareness is the main reason for preanalytical errors. This can be avoided by proper communication and coordination between laboratory and wards, proper training and continuing medical education programs for laboratory and paramedical staffs, and knowledge of the intervening factors that can influence laboratory results.

Key words:

Hematology, inadequate samples, laboratory, preanalytical errors

\section{Introduction}

$\neg$ he modern-day diagnosis is mainly L dependent on reliable laboratory data. There is a drastic improvement in the performance of laboratories due to remarkable advances in automation, sample collection, transport, and dispatch of reports. ${ }^{[1]}$ Medical laboratories also play a vital role in the decision-making of physicians about their patients. About $60 \%-70 \%$ of clinical decisions regarding admission, prescription, and discharge are based on laboratory results.

This is an open access article distributed under the terms of the Creative Commons Attribution-NonCommercial-ShareAlike 3.0 License, which allows others to remix, tweak, and build upon the work non-commercially, as long as the author is credited and the new creations are licensed under the identical terms.

For reprints contact: reprints@medknow.com
Since they play a significant role, more importance should be given to the quality of laboratory tests. ${ }^{[2]}$ However, errors can occur in any phase during the processing of blood sample. The errors in laboratory practice are classified into preanalytical, analytical, and postanalytical phase depending on the time of presentation. ${ }^{[3-5]}$

An important component of laboratory medicine is preanalytical phase. ${ }^{[5]}$ The preanalytical phase comprises all the processes occurring before the sample being processed in the laboratory. ${ }^{[6]}$ It includes

How to cite this article: Arul P, Pushparaj M, Pandian K, Chennimalai L, Rajendran K, Selvaraj E, et al. Prevalence and types of preanalytical error in hematology laboratory of a tertiary care hospital in South India. J Lab Physicians 2018;10:237-40. 
specimen collection, handling and processing variables, physiological variables, and endogenous variables. Certain preanalytical variables, namely, specimen variables can be controlled; whereas knowledge of uncontrollable variables needs to be well understood in order to be able to separate their effects from disease-related changes affecting laboratory results. ${ }^{[4,7]}$

Errors occurred during preanalytical phase was $61.9 \%$. The reported types of preanalytical error are ordering tests on the wrong patient, misidentifying the patient, ordering the wrong test, missing sample and/or test request, wrong or missing identification, contamination from infusion route, hemolyzed, clotted, and insufficient samples, inappropriate containers, improper labeling of containers, inappropriate blood-to-anticoagulant ratio, and inappropriate transport and storage conditions. ${ }^{\left[{ }^{[8,9]}\right.}$ The laboratories have to bear the burden of the inconsistencies or incorrect reporting that can ensue because of these preanalytical errors. ${ }^{[6]}$ Analytical errors can be minimized with the recent advancement in technology and introduction of automation in hematology laboratories provided good quality control practices are followed..$^{[10]}$ The present study was undertaken to find the prevalence and types of preanalytical errors at a tertiary care hospital in South India.

\section{Materials and Methods}

This cross-sectional study was carried out in Haematology Laboratory, Department of Pathology, at our institution over a period of 1 year between June 2016 and May 2017 after obtaining approval from the Institutional Ethical Committee. Blood samples collected in vacutainers during this period were included in the study. Blood collection for outpatient department (OPD) was centralized (central blood collection center) for different sections of central laboratory which cater the samples to various sections such as hematology, clinical pathology, biochemistry, and microbiology. OPD phlebotomies were performed by laboratory technologist, whereas blood samples from inpatients' department (IPD) were collected by staff nurses. The samples from IPD and OPD (central blood collection center) were delivered to the hematology laboratory by paramedical staff and laboratory support staff, respectively.

A total of 118,732 samples were received in hematology laboratory, of which 62,474 were from OPD and 56,258 were from IPD. These samples were analyzed for following preanalytical variables: (a) Misidentification (incorrectly labeled vials or incorrectly filled forms), (b) incorrect vials (wrong choice of vials), (c) inadequate samples, (d) clotted samples, (e) diluted samples, and (f) hemolyzed samples.
Master charts were prepared and sum of errors was calculated. Their relative frequencies when compared with the total specimens were also calculated and presented as percentage. All the statistical analyses were performed using International Business Machines (IBM) Corporation Statistical Package for the Social Sciences (SPSS) Statistics for Windows (version 20.0. IBM Corporation, Armonk, New York, USA).

\section{Results}

A total of 118,732 samples containing 62,474 OPD and 56,258 IPD were analyzed for preanalytical errors based on the above-mentioned parameters. The overall prevalence of preanalytical errors found was 513 samples, which is $0.43 \%$ of the total number of samples received. Frequencies of preanalytical errors in both OPD and IPD are shown in Tables 1 and 2. The most common preanalytical error was inadequate samples followed by clotted samples. Overall frequencies (OPD and IPD) of preanalytical errors such as misidentification, incorrect vials, inadequate samples, clotted samples, diluted samples, and hemolyzed samples were $0.02 \%, 0.05 \%$, $0.2 \%, 0.12 \%, 0.02 \%$, and $0.03 \%$, respectively.

\section{Discussion}

Modern methods have been applied in medical laboratory to reduce the errors at preanalytical, analytical, and postanalytical phases of sample processing. ${ }^{[11]}$ However, errors are more commonly found in pre- and postanalytical phases than that in analytical phase. The major cause of the problem is that some phases are not in

Table 1: Frequency of the various preanalytical errors observed in outpatient department samples $(n=62,474)$

\begin{tabular}{lc}
\hline Preanalytical errors & Number of samples (\%) \\
\hline Misidentification & $9(0.01)$ \\
Incorrect vials & $28(0.04)$ \\
Inadequate samples & $104(0.17)$ \\
Clotted samples & $67(0.11)$ \\
Diluted samples & 0 \\
Hemolyzed samples & $15(0.02)$ \\
Total & $223(0.35)$ \\
\hline
\end{tabular}

Table 2: Frequency of the various preanalytical errors observed in inpatient department samples $(n=56,258)$

\begin{tabular}{lc}
\hline Preanalytical errors & Number of cases $(\%)$ \\
\hline Misidentification & $14(0.02)$ \\
Incorrect vials & $32(0.06)$ \\
Inadequate samples & $131(0.23)$ \\
Clotted samples & $72(0.13)$ \\
Diluted samples & $23(0.04)$ \\
Hemolyzed samples & $18(0.03)$ \\
Total & $290(0.52)$ \\
\hline
\end{tabular}


direct control of the laboratory personnel. ${ }^{[12]}$ Preanalytical errors are largely being caused by human mistakes and the majority of these errors are preventable..$^{[8,13,14]}$ This is comprehensible since the preanalytical phase involves much more human handling compared to the analytical and postanalytical phases..$^{[15]}$

Use of automated analyzers in preanalytical phase has also helped to minimize the laboratory errors. Introduction of automated robotic workstations at preanalytical stage further reduces the hazards and errors. ${ }^{[1]}$ Computerized order entry simplifies test ordering and eliminates a second person from transcribing the orders. Automated phlebotomy tray preparation provides a complete set of labeled blood tubes and labels for hand labeling in a single tray for each patient. Preanalytical robotic workstations automate some of the steps and reduce the number of manual steps involving more people. Barcodes also simplify specimen routing and tracking. ${ }^{[16]}$

In the current study, preanalytical errors were more common in IPD samples. That might be due to the grave conditions of hospitalized patients and a variety of staff involved in the total testing processes. ${ }^{[2]}$ Nurses/paramedical staff collected the samples in IPD and many of them were not aware of the importance of sample collection by proper techniques. This could be the reason for higher percentage of preanalytical errors in samples collected from IPD of this study. Hence, nurses / paramedical staff need to be more specialized in taking blood samples and other interventions.

Inadequate samples $(0.2 \%)$ accounted for the majority of errors in the present study. This could be due to ignorance of phlebotomists, difficult sampling in pediatrics, patients with chronic debilitating diseases, and patients on chemotherapy whose thin veins are difficult to localize. ${ }^{[6]}$ There is a risk of cell shrinkage and low mean corpuscular volume when less volume of blood is withdrawn than the recommended volume to tubes containing ethylenediaminetetraacetic acid (EDTA). ${ }^{[17]}$

The second most common error in this study was clotted samples $(0.12 \%)$. Clotted samples can be easily detected; however, microclots are difficult to detect, particularly anticoagulated blood samples. The presence of clots in EDTA samples can be explained primarily due to increased blood-to-additive ratio (inadequate EDTA) or improper mixing of the sample after collection. ${ }^{[10,17]}$ In our study, clotted samples could be due to improper mixing.

Both misidentifications (incorrectly labeled vials or incorrectly filled forms) and incorrect vials (wrong choice of vials) constituted $0.07 \%$ in the present study. This could be due to excessive patient load or lack of awareness regarding patient information. ${ }^{[17]}$ These types of preanalytical errors can have serious adverse effects or can lead to completely wrong treatment of the patient. ${ }^{[18]}$

Diluted samples accounted for $0.02 \%$ in the current study and it was observed only in IPD where the samples can be diluted with intravenous (IV) fluids. Nursing staff sometimes fail to recognize the importance of using veins in which IV lines have not been introduced. ${ }^{[19]}$ It has been suggested that whenever IV fluid is being administered in a patient's arm, blood should be drawn from the opposite arm. If an IV fluid is running in both arms, sample may be drawn after IV infusion is turned off for at least $2 \mathrm{~min}$ before venipuncture and applying the tourniquet below the IV infusion site. ${ }^{[10]}$

A hemolyzed sample noted in our study was $0.03 \%$. Hemolysis of samples occurs in certain situations, namely, when blood is forced through a needle, shaking the tubes vigorously, and centrifuging the sample before clotting being formed. ${ }^{[20]}$ It also leads to a more turnaround time due to the need for fresh samples for processing. Detection of hemolyzed samples in hematology laboratories is somewhat difficult than that in biochemistry laboratories which can lead to falsely lower frequency of preanalytical error ${ }^{[6]}$

In the present study, the prevalence of preanalytical errors in hematology laboratory was $0.43 \%$ of the total samples. This observation was almost comparable to those of recently published Indian studies [Table 3] which confirm that problems directly related to specimen collection are the main cause of preanalytic errors. ${ }^{[6,10,19]}$ This can be reduced by competency check of staffs by conducting practical and theory assessment at regular

Table 3: Comparison of prevalence (\%) of preanalytical errors in the present study with various studies

\begin{tabular}{lcccc}
\hline Preanalytical errors & $\begin{array}{c}\text { Present study } \\
\text { OPD + IPD }\end{array}$ & $\begin{array}{c}\text { Upreti et al. }{ }^{[19]} \\
\text { OPD + IPD }\end{array}$ & $\begin{array}{c}\text { Chawla et al. }{ }^{[6]} \\
\text { OPD + IPD }\end{array}$ & $\begin{array}{c}\text { Narang et al. }{ }^{[10]} \\
\text { OPD + IPD }\end{array}$ \\
\hline Misidentification & 0.02 & 0.35 & 0.47 & 0.03 \\
Incorrect vials & 0.05 & 0.16 & 0.23 & 0.06 \\
Inadequate samples & 0.2 & 0.19 & - & - \\
Clotted samples & 0.12 & 0.13 & - & - \\
Diluted samples & 0.02 & 0.04 & 0.74 & - \\
Hemolyzed samples & 0.03 & 0.09 & 0.07 & - \\
Lipemic samples & - & - & & \\
\hline
\end{tabular}

OPD = Outpatient department, IPD = Inpatient department 
intervals and attending continuing medical education programs related to quality control in hematology.

The limitations of our study were that registration of the errors during the evening, night shifts, and holidays was not completely performed. Time between sample collection and actual analytic process was not calculated. More studies have to be carried out to examine differences between day and night shifts separately and at multicenters.

\section{Conclusion}

The present study concluded that incorrect phlebotomy techniques due to lack of awareness is the main reason for preanalytical errors. This can be avoided by proper communication and coordination between laboratory and wards, proper training and continuing medical education programs for laboratory and paramedical staffs, and knowledge of the intervening factors that can influence laboratory results.

\section{Financial support and sponsorship Nil.}

\section{Conflicts of interest}

There are no conflicts of interest.

\section{References}

1. Boone DJ. Governmental perspectives on evaluating laboratory performance. Clin Chem 1993;39:1461-5.

2. Abdollahi A, Saffar H, Saffar H. Types and frequency of errors during different phases of testing at a clinical medical laboratory of a teaching hospital in Tehran, Iran. N Am J Med Sci 2014;6:224-8.

3. Aakre KM, Langlois MR, Watine J, Barth JH, Baum H, Collinson P, et al. Critical review of laboratory investigations in clinical practice guidelines: Proposals for the description of investigation. Clin Chem Lab Med 2013;51:1217-26.

4. Chhillar N, Khurana S, Agarwal R, Singh NK. Effect of pre-analytical errors on quality of laboratory medicine at a neuropsychiatry institute in North India. Indian J Clin Biochem 2011;26:46-9.

5. Narayanan S. The preanalytic phase. An important component of laboratory medicine. Am J Clin Pathol 2000;113:429-52.

6. Chawla R, Goswami V, Tayal D, Mallika V. Identification of the types of preanalytical errors in the clinical chemistry laboratory: 1-year study of G.B. Pant Hospital. Lab Med 2010;41:89-92.

7. Rana SV. No preanalytical errors in laboratory testing: A beneficial aspect for patients. Indian J Clin Biochem 2012;27:319-21.

8. Carraro P, Plebani M. Errors in a stat laboratory: Types and frequencies 10 years later. Clin Chem 2007;53:1338-42.

9. Hammerling JA. A review of medical errors in laboratory diagnostics and where we are today. Lab Med 2012;43:41-4.

10. HarsimranKaur VN, Selhi PK, Sood N, Singh A. Preanalytical errors in hematology laboratory- an avoidable incompetence. Iran J Pathol 2016;11:151-4.

11. Plebani M. Errors in clinical laboratories or errors in laboratory medicine? Clin Chem Lab Med 2006;44:750-9.

12. Mohammedsaleh ZM, Mohammedsaleh F. A review article of the reduce errors in medical laboratories. Glob J Health Sci 2014;7:46-51.

13. Kalra J. Medical errors: Impact on clinical laboratories and other critical areas. Clin Biochem 2004;37:1052-62.

14. Astion ML, Shojania KG, Hamill TR, Kim S, Ng VL. Classifying laboratory incident reports to identify problems that jeopardize patient safety. Am J Clin Pathol 2003;120:18-26.

15. Rynning M, Wentzel-Larsen T, Bolann BJ. A model for an uncertainty budget for preanalytical variables in clinical chemistry analyses. Clin Chem 2007;53:1343-8.

16. Da Rin G. Pre-analytical workstations: A tool for reducing laboratory errors. Clin Chim Acta 2009;404:68-74.

17. Ashavaid TF, Dandekar SP, Khodaiji S, Ansari MH, Singh AP. Influence of method of specimen collection on various preanalytical sample quality indicators in EDTA blood collected for cell counting. Indian J Clin Biochem 2009;24:356-60.

18. Favaloro JE, Funk DM, Lippi G. Preanalytical variables in coagulation testing associated with diagnostic errors in hemostasis. Lab Med 2012;43:1-10.

19. Upreti S, Upreti S, Bansal R, Jeelani N, Bharat V. Types and frequency of preanalytical errors in haematology lab. J Clin Diagn Res 2013;7:2491-3.

20. Carraro P, Servidio G, Plebani M. Hemolyzed specimens: A reason for rejection or a clinical challenge? Clin Chem 2000;46:306-7. 